\title{
EHMTI-0277. Monitoring the use of symptomatic drugs in headache patients: a 6 month follow-up
}

\author{
C Voiticovschi-losob ${ }^{1 *}$, F Antonaci ${ }^{2}$, L Gervasio ${ }^{3}$, C Fattore $^{4}$, M Bianchi ${ }^{5}$, I De Cillis ${ }^{6}$, G Nappi ${ }^{7}$, N Vanacore \\ From 4th European Headache and Migraine Trust International Congress: EHMTIC 2014 \\ Copenhagen, Denmark. 18-21 September 2014
}

\section{Background}

Headache is an extremely common neurological problem. Italy is the first European country for OTC consumption with related problems of self-medication and risk of medication overuse headache (MOH).

\section{Aim}

to monitor the consumption of symptomatic drugs for headache and to prevent drugs abuse/dependence.

\section{Materials and methods}

274 patients using symptomatic drug for headache were recruited in 32 pharmacies in the Pavia Health District. A telephonic interview was carried out in 199 patients; 179 entered the study at baseline (T0) and $112(22 \mathrm{M}$ and $90 \mathrm{~F}$, mean age $45.0 \pm 11.5 \mathrm{yrs}$.) were followed-up at 6 months (T6).

\section{Results}

patients with chronic migraine or $\mathrm{MOH}$ at $\mathrm{T} 0$ were 39 and 7 at T6. Days/month with headache at T6 vs T0 were $4.3 \pm 0.6$ vs $9.7 \pm 0.8(\mathrm{p}<0.0001)$. Attacks/month at T6 vs T0 were slightly reduced $(1.9 \pm 0.2$ vs $7.6 \pm 0.8 \mathrm{p}=$ $0.09)$. A significant decrease of the doses of analgesics consumption $/$ month was noted $(\mathrm{T} 6=13.2 \pm 1.2$ vs $\mathrm{T} 0=$ $17.0 \pm 2.2, \mathrm{p}=0.013)$. An increase in quality of life was found on MIDAS scores at T6 vs T0 (13.4 \pm 1.8 vs $23.7 \pm$ $2.5 ; \mathrm{p}=0.00)$ and in the quality of treatment received $($ HURT $)(5.6 \pm 0.4$ vs $9.9 \pm 0.5 ; \mathrm{p}=0.00)$.

\section{Conclusions}

Our results highlight that the change from self medication to medical care may reduce the numbers of symptomatic treatment, the headache days/ month and ameliorate the quality of life in patients with headache. A longer follow-up (i.e. 12 month) may provide further evidence on improvement of the clinical picture of headache patients and prevention of $\mathrm{MOH}$.

No conflict of interest.

\section{Acknowledgments}

Research grant from the Italian Ministry of Health (2013) to IRCCS C. Mondino.

\section{Authors' details}

${ }^{1}$ Headache Center, C. Mondino National Institute of Neurology Foundation IRCCS Pavia Italy and State Medical and Pharmaceutical University "NicolaeTestemitanu", Chisinau, Moldova. ${ }^{2}$ Headache Center, C. Mondino National Institute of Neurology Foundation IRCCS and Dept. of Brain and Behavioral Sciences University of Pavia, Pavia, Italy. ${ }^{3}$ Pharmaceutic Service, C. Mondino National Institute of Neurology Foundation IRCCS, Pavia, Italy. ${ }^{4}$ Clinical Trial Center and Antiepileptic Drugs, C. Mondino National Institute of Neurology Foundation IRCCS, Pavia, Italy. ${ }^{5}$ Headache Center, C. Mondino National Institute of Neurology Foundation, Pavia, Italy. ${ }^{6}$ Headache Center, C. Mondino National Institute of Neurology Foundation IRCCS, Pavia, Italy. ${ }^{7}$ Headache Center, C. Mondino National Institute of Neurology Foundation IRCCS and Dept. of Brain and Behavioral Sciences, Pavia, Italy. ${ }^{8}$ Statistics, Istituto Superiore di Sanità, Roma, Italy.

Published: 18 September 2014

doi:10.1186/1129-2377-15-S1-D74

Cite this article as: Voiticovschi-losob et al: EHMTI-0277. Monitoring the use of symptomatic drugs in headache patients: a 6 month follow-up. The Journal of Headache and Pain 2014 15(Suppl 1):D74. 